\title{
Morphogenesis-regulated localization of protein kinase A to genomic sites in Candida albicans
}

\author{
Alida Schaekel ${ }^{1,2}$, Prashant R Desai ${ }^{1}$ and Joachim F Ernst ${ }^{1,2^{*}}$
}

\begin{abstract}
Background: The human fungal pathogen Candida albicans is able to undergo morphogenesis from a yeast to a hyphal growth form. Protein kinase A (PKA) isoforms Tpk1 and Tpk2 promote hyphal growth in a signalling pathway via the transcription factor Efg1.

Results: C. albicans strains producing epitope-tagged Tpk1 or Tpk2 were used in genome-wide chromatin immunoprecipitation on chip (ChIP chip) to reveal genomic binding sites. During yeast growth, both PKA isoforms were situated primarily within ORFs but moved to promoter regions shortly after hyphal induction. Binding sequences for Tpk2 greatly exceeded Tpk1 sites and did not coincide with binding of the PKA regulatory subunit Bcy1. Consensus binding sequences for Tpk2 within ORFs included ACCAC and CAGCA motifs that appeared to bias codon usage within the binding regions. Promoter residency of Tpk2 correlated with the transcript level of the corresponding gene during hyphal morphogenesis and occurred near Efg1 binding sites, mainly on genes encoding regulators of morphogenesis.
\end{abstract}

Conclusions: PKA isoforms change their genomic binding sites from ORF to promoter regions during yeast-hyphal morphogenesis. Tpk2 binds preferentially to promoters of genes encoding regulators of cellular morphogenesis.

Keywords: Candida albicans, Protein kinase A, Tpk1, Tpk2, Efg1, Morphogenesis

\section{Background}

The fungus Candida albicans is an important cause of human disease, causing tenacious superficial and lifethreatening systemic infections. Its virulence depends to a large extent on its ability to switch between a yeast and a hyphal growth form [1]. Environmental conditions favouring hyphal development include molecules of the human host acting as inducers, as well as physical parameters such as body temperature. Protein kinase A (PKA) isoforms Tpk1 and Tpk2 have crucial roles as signalling kinases because they mediate several adaptation responses to host contact [2-4]. In inducing conditions, cAMP is generated by adenylate cyclase (Cyr1) and triggers PKA activity by binding and removal of the inhibitory subunit Bcy1, which associates with Tpk1 and Tpk2 [5-7]. The cAMP-PKA pathway subsequently activates the Efg1 transcription factor, which represents the

\footnotetext{
* Correspondence: joachim.ernst@uni-duesseldorf.de

'Department Biologie, Molekulare Mykologie, Heinrich-Heine-Universität, Düsseldorf, Germany

${ }^{2}$ Manchot Graduate School Molecules of Infection, Heinrich-Heine-Universität, Düsseldorf, Germany
}

(c) 2013 Schaekel et al.; licensee BioMed Central Ltd. This is an open access article distributed under the terms of the Creative Commons Attribution License (http://creativecommons.org/licenses/by/2.0), which permits unrestricted use, distribution, and reproduction in any medium, provided the original work is properly cited.

central hub controlling downstream events including morphogenesis and metabolic adaptation [1,8-10]. Efg1 fulfills its morphogenetic functions by association with co-regulators Czf1, Flo8, Slf1 and Slf2 [11,12]. Interestingly, in spite of their association with the same regulator protein Bcyl, both PKA isoforms exert specific environment-dependent functions with regard to hyphal morphogenesis [3] and Tpk2 but not the Tpk1 isoform mediates downregulation of EFG1 expression early in hyphal induction [13].

PKA localization differs among species: in budding yeast the PKA holoenzyme is localized in the nucleus [14], whereas in fission yeast it resides in the cytoplasm [15] and in mammalian cells PKA catalytic subunits bind to anchoring proteins in different intracellular localizations $[16,17]$. In spite of these differences, it appears that in all species important AGC kinase activities are needed in the nucleus. Increased cAMP levels lead to partial entry of PKA catalytic subunits into nuclei of fission yeast [15] and mammalian cells $[16,17]$. In C. albicans, phosphorylation of the Tpk2 target protein Efg1 is likely to occur in the 
nucleus since Efg1 has been detected exclusively in the nucleus [18]. In Saccharomyces cerevisiae, activated PKA and the mitogen-activated protein kinase (MAPK) Hog1 were found to associate with promoters and coding regions of genes regulated by these kinases [19-21]. Action of the Hog1 MAPK on the Sko1 trancriptional repressor required the activity of kinase Sch9, which is structurally related to PKA, on the promoters of target genes [22]. The latter findings suggested that also in C. albicans, Tpk isoforms and possibly other kinases reside on genes that represent downstream targets of PKA signalling during hyphal morphogenesis. In this study, we strengthen this concept by demonstrating that PKA isoforms reside on specific genomic locations that change dramatically during morphogenesis from ORF to promoter regions. During the yeast-hyphal transition, genomic Tpk2 binding sites identify genes with known functions in dimorphism and suggest the identity of new genes involved in this cellular differentiation process.

\section{Results and discussion}

\section{C. albicans strains producing HA-tagged PKA kinases}

The single remaining allele encoding the catalytic subunits of PKA kinase was modified in heterozygous C. albicans mutants to add sequences specifying a C-terminal triple hemagglutinin (HA) epitope tag. Immunoblotting revealed the presence of HA-fusions to Tpk1 and Tpk2 proteins in cellular extracts of these strains during yeast growth (Figure 1A). Immunofluorescence microscopy revealed that the majority of fusion proteins resided in the cytoplasm of cells, while a minor fraction of $\mathrm{Tpk} 2{ }^{\mathrm{HA}}$ was also detectable at the inside rim of the nucleus (Figure 1B, yellow dots). Similar Tpk cellular localization was observed in cells that were briefly (30 min) induced by $10 \%$ serum to form hyphae (data not shown).

To verify that HA fusion proteins were functional in the constructed strains we tested their filamentous growth, which is known to be regulated by the activity of Tpk1 and Tpk2 proteins [2,3]. Inactivation of a single TPK1 allele abolishes hyphal growth [3] but the TPK1 ${ }^{H A}$ / TPK1 transformant formed hyphae as the wild-type strain (Figure $1 \mathrm{C}$ ) indicating that the $\mathrm{Tpk} 1^{\mathrm{HA}}$ fusion protein is functional. Both TPK2 alleles need to be inactivated to prevent hyphal growth [2]; therefore, it was verified that the filamentation phenotype of the $T P K 2^{H A} / t p k 2$ strain mimicked the TPK2/tpk2 strain but not the $t p k 2 / t p k 2$ homozygous mutant (Figure $1 C$ ). This result shows that the Tpk $2^{\mathrm{HA}}$ fusion protein is functional. In summary, use of HA-tagged Tpk proteins revealed that in C. albicans as in fission yeast [15] the majority of PKA is located in the cytoplasm. The exclusive localization of a possibly non-functional Tpk1-GFP fusion within the nucleus [5] was not confirmed by the HA-tagged Tpk1 protein.

\section{A}

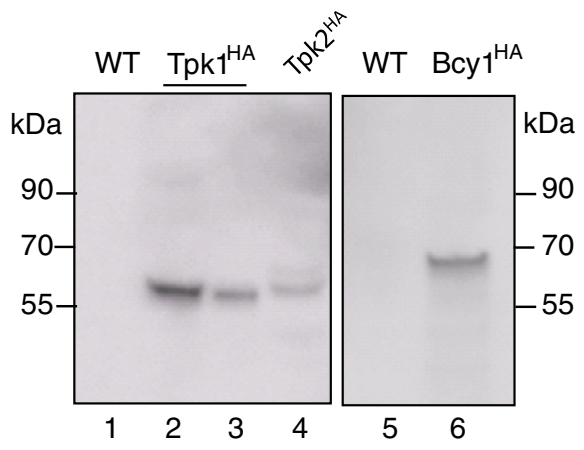

B

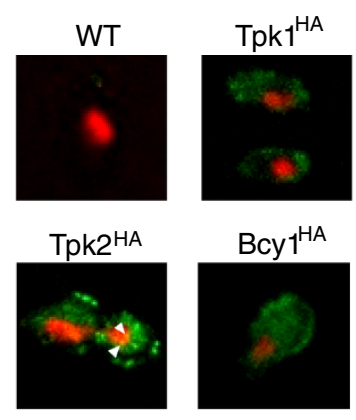

C
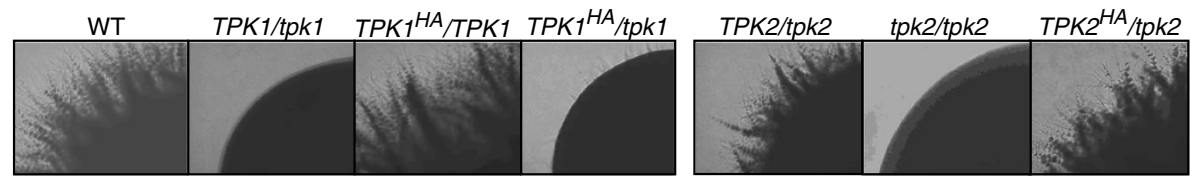

Figure 1 Epitope-tagged PKA in C. albicans. A. Immunoblotting to detect HA-tagged PKA subunits. Strains were grown in liquid YPD medium to $\mathrm{OD}_{600}=0.6-0.8$ and proteins in cell extracts $(50 \mu \mathrm{g})$ were separated by SDS-PAGE (10\% acrylamide), blotted onto PVDF membranes and probed using anti-HA antibody. Strains tested were CAF2-1 (WT: lanes 1, 5), AF1003 (TPK1 $1^{\text {HA }}$ : lane 2), AF1004 (TPK1 ${ }^{\text {HA }}$ : lane 3), AF1005 (TPK2 ${ }^{\text {HA }}$ : lane 4) and AF1007 (BCY ${ }^{\text {HA }}$ : lane 6). B. Fluorescence microscopy of transformant cells treated with rat anti-HA antibody, which was detected with FITC-labelled anti-rat antibody (green signal) and DAPI to detect nuclei (red signal); yellow overlay signals are indicated by white triangles. C. Filament formation of strains producing HA-tagged Tpk proteins. Strains CAF2-1 (wt), II (TPK1/tpk1), TPK7 (TPK2/tpk2), TPO7.4 (tpk2/tpk2), AF1003 (TPK1 HA/TPK1), AF1004 $\left(T P K 1^{H A} / t p k 1\right)$ and AF1005 (TPK2 $\left.{ }^{H A} / t p k 2\right)$ were grown for $3 \mathrm{~d} / 37^{\circ} \mathrm{C}$ on Spider agar and single colonies were photographed. 


\section{Genomic localization of Tpk proteins}

The above results suggested that a minor fraction of cellular PKA catalytic subunits resides in the nucleus of C. albicans cells. Furthermore, the presence of PKA isoforms and other kinases at target genes had been demonstrated previously in S. cerevisiae [19-21]. To verify, if nuclear PKA isoforms bind specific genomic targets in C. albicans we performed ChIP chip experiments with strains containing HA-tagged PKA isoforms; strains producing authentic non-tagged Tpk proteins were used as reference strains. Tpk1 and Tpk2 localization was examined during yeast growth or alternatively, following a brief period (30 min) of hyphal induction by $10 \%$ serum. During this time period, early regulatory processes take place that reprogram cells to allow hyphal growth. This became evident in wild-type cells by the formation of germ tubes after 30-60 min of induction.

A significant number of Tpk genomic binding sites was identified in both yeast and hyphal cells in duplicate ChIP chip experiments (see Additional file 1: Tables S1-S4). About tenfold more Tpk2 than Tpk1 binding sites were detected during yeast growth but Tpk1 binding increased during hyphal induction. A particular importance of the Tpk2 isoform for C. albicans morphogenesis under liquid growth conditions was reported previously [3]. The most significant peaks, which were overlapping in both replicates, were defined as binding sites. These sites were associated with ORF and promoter sequences of target genes (promoter binding sites within two divergently transcribed genes were assigned to both genes). Analyses indicated that very rarely, both Tpk1 and Tpk2 bind to same gene (Figure 2A). An exception is $\mathrm{HGCl}$ encoding an important regulator of hypha formation [23], which bound both Tpk1 and Tpk2 during hyphal induction. This result indicates that both catalytic PKA isoforms have mostly different genomic targets.

Importantly, during yeast growth both Tpk1 and Tpk2 were bound mostly within ORFs of target genes, while hyphal induction reduced ORF binding and favoured promoter binding or joint promoter-ORF binding (Figure 2B). Under yeast and hyphal growth conditions, all Tpk1 target genes and the majority of Tpk2 target genes were different (Figure 2C). Thus, ORF-to-promoter switching rarely occurs on the same gene during yeast-hyphal morphogenesis.

Growth of C. albicans in rich medium does not trigger hyphal formation, because PKA activity is repressed by the regulatory PKA subunit Bcy1 [5]. To test if during yeast growth both Tpk isoforms and Bcy1 bind to ORFs a ChIP chip experiment was performed on a $B C Y 1^{H A}$, $B C Y 1$ strain (AF1007). Immunoblotting showed the production of HA-tagged Bcy1 in transformants (Figure 1A) and immunofluorescence demonstrated that this protein resides mainly in the cytoplasm (Figure 1B). The $\mathrm{Bcy}^{\mathrm{HA}}$ fusion produced by transformants is functional because the $B C Y 1^{H A} / B C Y 1$ strain was insensitive to heat shock $\left(2 \mathrm{~h}\right.$ at $\left.50^{\circ} \mathrm{C}\right)$ (data not shown), unlike a $B C Y 1 / b c y 1$ strain [4]. Data analyses revealed a moderate number of genomic Bcy1 binding sites during yeast growth (see Additional file 1: Table S5). However, binding sites did not coincide to a great extent with Tpk1 or Tpk2 binding sites, either during yeast growth or during hyphal induction (Figure 2C). These results indicate that in general, Tpk1 or Tpk2 isoforms do not bind to target ORFs in the form of Tpk-Bcy1 holoenzyme complexes. Conceptually, ORF-bound Tpk proteins could either be active because of the absence of Bcy1 or their activities may be regulated by yet unknown mechanisms.

\section{Gene ontology analysis of PKA binding sites}

Gene ontology (GO) analyses [24] of genes close to or harbouring PKA binding sites revealed significant GO terms for Tpk2 kinase but not for the Tpk1 isoform in any growth condition. During yeast growth, Tpk2 bound preferentially to ORFs (or both ORF and promoters) of genes encoding components of the general transcription machinery (e. g. Asf1, Def1, Swil, Spt6), in addition to ORFs encoding transcription factors involved in carbon source utilization (e. g. Rca1, Snf5, Tup1) or filamentous growth (e. g. Efg1, Tup1, Sfl1) (Figure 3A). Furthermore, ORFs of genes for components of signalling pathways leading to hyphal formation (e. g. Cek1, Msb2) were bound by Tpk2 during yeast growth (Figure 3A). During hyphal induction, Tpk2 bound mostly to promoter regions of genes involved in filamentous growth (40 genes including HGC1, RAS1) (Figure 3B). Tpk2 target sites included promoters of genes encoding 20 transcription factors directly binding DNA (mostly containing zinc finger motifs). In summary, Tpk2 genomic binding shows a distinctive pattern of binding to morphogenesis-related genes, specifically the binding to ORFs during yeast growth and to promoters during hyphal induction. Tpk2 target genes are often bound also by Efg1 $[13,25,26]$ in promoter regions or by the Set3C histone deacetylase complex [27] within their ORFs (superscripts d, e, in Figure 3). Recently, direct binding of heat shock factor-type transcriptional regulators Sfl1 and Sfl2 to Efg1 has been demonstrated [12], a finding also associating the genomic localization of these regulators with Tpk2 binding sites. In summary, Tpk2 binding identifies genes with known functions in filamentous growth and predicts such functions for yet uncharacterized C. albicans genes (designated ORF19. in Figure 3).

\section{ORF binding during yeast growth}

ORF binding by PKA isoforms occurred mainly during yeast growth and included two relevant $C$. albicans genes, EFG1 and MSB2. EFG1 is required for the initial phase of yeast-hyphal transition but it is downregulated rapidly 
A

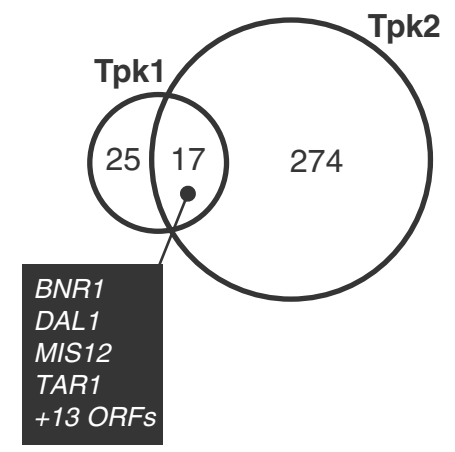

Hypha

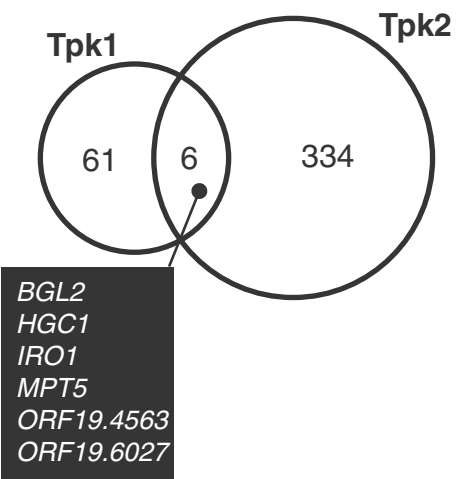

B

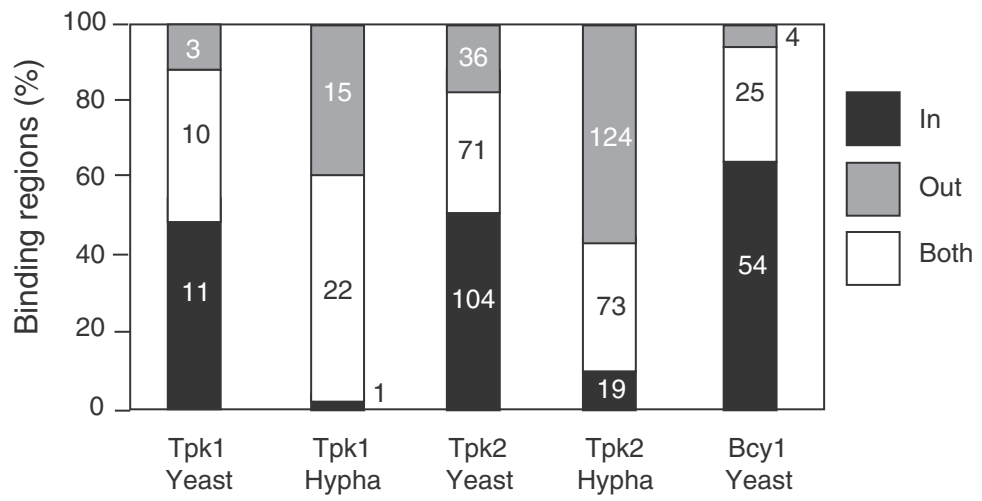

C

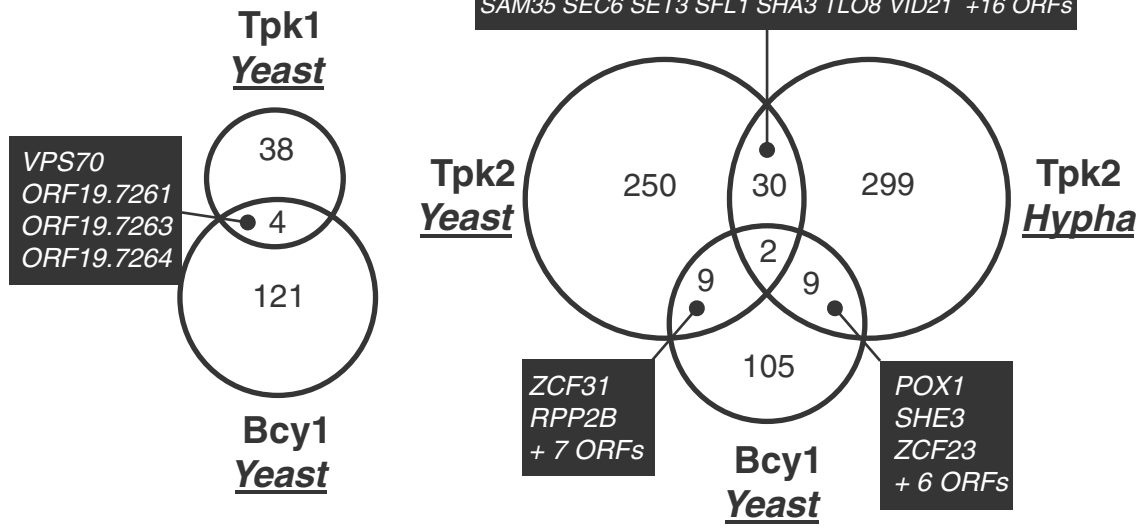

Figure 2 Genomic localization of PKA subunits. A. Genomic binding of PKA subunits. Venn diagrams number genes containing Tpk1 or Tpk2 binding sites in their promoter or ORF sequences; cells were grown in the yeast form or induced 30 min for hypha formation using 10\% horse serum. Names of genes in the Tpk1/Tpk2 intersection are listed. B. Genomic PKA subunit localization. For each subunit, numbers of binding sites inside an ORF (black box), outside an ORF (promoter) (grey box) or at both sites (white box) are listed. C. Genomic binding of Tpk1, Tpk2 and Bcy1 subunits. The Venn diagram numbers indicate genes associated with the Tpk1 or Tpk2 catalytic subunit under yeast growth or hyphal induction, as well as the localization of the Bcy1 regulatory subunit during yeast growth. Gene names in the intersections are indicated.

by negative autoregulation to allow undisturbed morphogenesis [29]; downregulation requires the Tpk2 but not the Tpk1 PKA isoform [13]. MSB2 encodes a membrane sensor for environmental cues leading to hypha formation via the Cek1 MAPK and its shed domain provides resistance to antimicrobial peptides [30,31].

During yeast growth, Tpk2 but not Tpk1 was bound to the EFG1 ORF. Binding was also observed to the 


\begin{tabular}{|c|c|c|c|}
\hline \multirow{2}{*}{$\begin{array}{l}\text { GO term } \\
\text { (genome frequency) }\end{array}$} & \multicolumn{3}{|l|}{ Tpk2 binding genes annotated to the term ${ }^{2}$} \\
\hline & $\overline{\text { Name }}$ & Frequency $^{3}$ & $P$ value $^{4}$ \\
\hline \multicolumn{4}{|l|}{ A. Yeast growth } \\
\hline Positive regulation of biological process ( $7.8 \%$ ) & 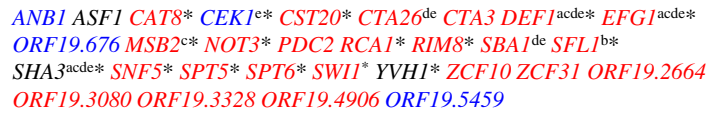 & $15.3 \%$ & 0.0322 \\
\hline Positive regulation of macromolecule metabolic process (3.49\%) & 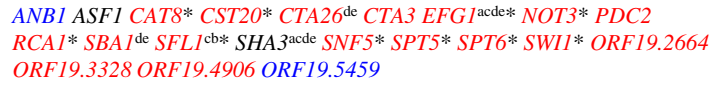 & $10.71 \%$ & 0.0375 \\
\hline $\begin{array}{l}\text { Carbon catabolite regulation of transcription from RNA polymerase II } \\
\text { promoter }(0.4 \%)\end{array}$ & CAT8* RCA $*$ SNF5* SPT6* SWII*TUPI $1^{\text {bde } *}$ & $3 \%$ & 0.0788 \\
\hline \multicolumn{4}{|l|}{ B. Hyphal induction } \\
\hline Growth $(9.6 \%)$ & 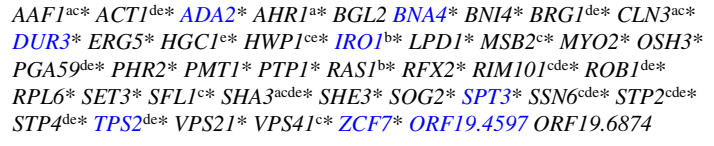 & $19.3 \%$ & $4.93 \mathrm{e}-06$ \\
\hline Filamentous growth $(9.3 \%)$ & 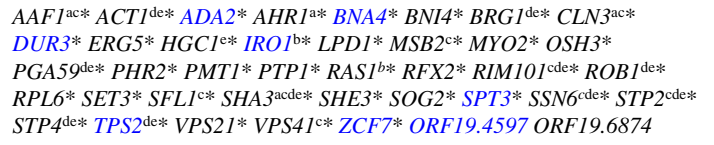 & $18.4 \%$ & $1.63 \mathrm{e}-05$ \\
\hline Cell communication $(7.9 \%)$ & 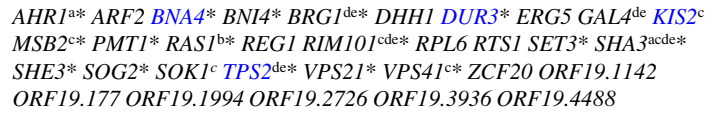 & $14.7 \%$ & 0.0132 \\
\hline Regulation of filamentous growth (2.6\%) & 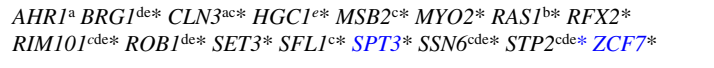 & $7.3 \%$ & 0.0119 \\
\hline Multi-organism process $(6.5 \%)$ & 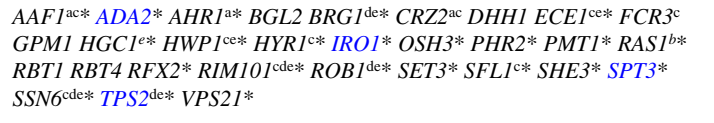 & $13.8 \%$ & 0.0280 \\
\hline Nucleic acid binding transcription factor activity $(3.4 \%)$ & 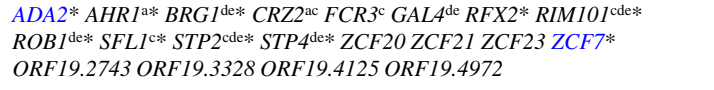 & $9.2 \%$ & 0.0003 \\
\hline $\begin{array}{l}\text { Sequence-specific DNA binding RNA polymerase II transcription factor } \\
\text { activity }(1.9 \%)\end{array}$ & 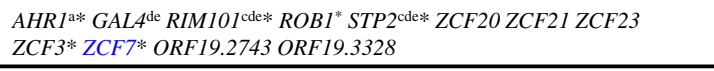 & $5.5 \%$ & 0.0089 \\
\hline \multicolumn{4}{|c|}{ 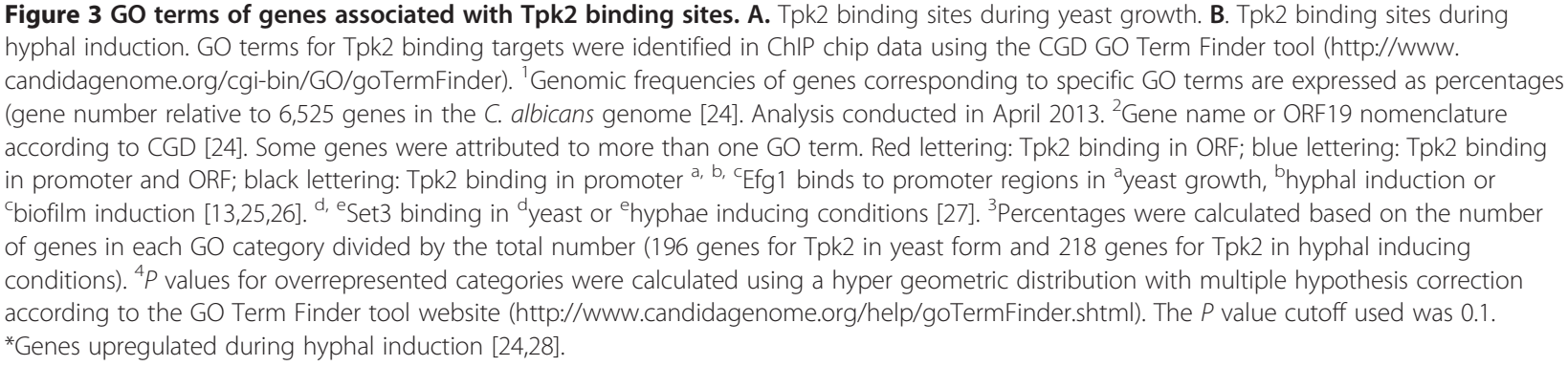 } \\
\hline
\end{tabular}

upstream gene (ORF19.612) but not to the downstream gene (ORF19.607) of EFG1 (Figure 4A). Similarly, Tpk2 but not Tpk1 bound the MSB2 ORF in a distinct peak (Figure 4B, a). Comparisons of MSB2 transcript levels in a wild-type strain and a homozygous tpk2 mutant, grown in the yeast form, revealed no significant differences (Figure 4B, c). Similarly, EFG1 promoter activity did not differ significantly between a wild-type and a $t p k 2$ mutant strain [13]. We conclude that $\mathrm{Tpk} 2$ residency at both target loci has no major influence on transcription/ transcript levels, although subtle regulatory influences on gene expression cannot be excluded. A function of Tpk2 binding at ORFs is suggested by a distinct codon preference in the Tpk2 binding region (see below).

\section{Promoter binding during hyphal induction}

During hyphal induction PKA isoforms bound preferentially to promoter regions. At the EFG1 promoter extensive binding of Tpk1 but not of Tpk2 was detected (Figure 4A). The broad Tpk1 binding area ranges from the EFG1 transcriptional start site through 1169 bp untranslated upstream sequences and ends close to the 3' end of the EFG1 ORF. The Tpk1 binding area matches one of the major binding sites for Efg1 in the yeast form indicating that shortly after hyphal induction, Tpk1 binding occurs concomitant with the release of Efg1 [13]. EFG1 promoter downregulation had been also observed in a tpk1 mutant [13] suggesting that Tpk1 has no major role in negative autoregulation of EFG1. 
A

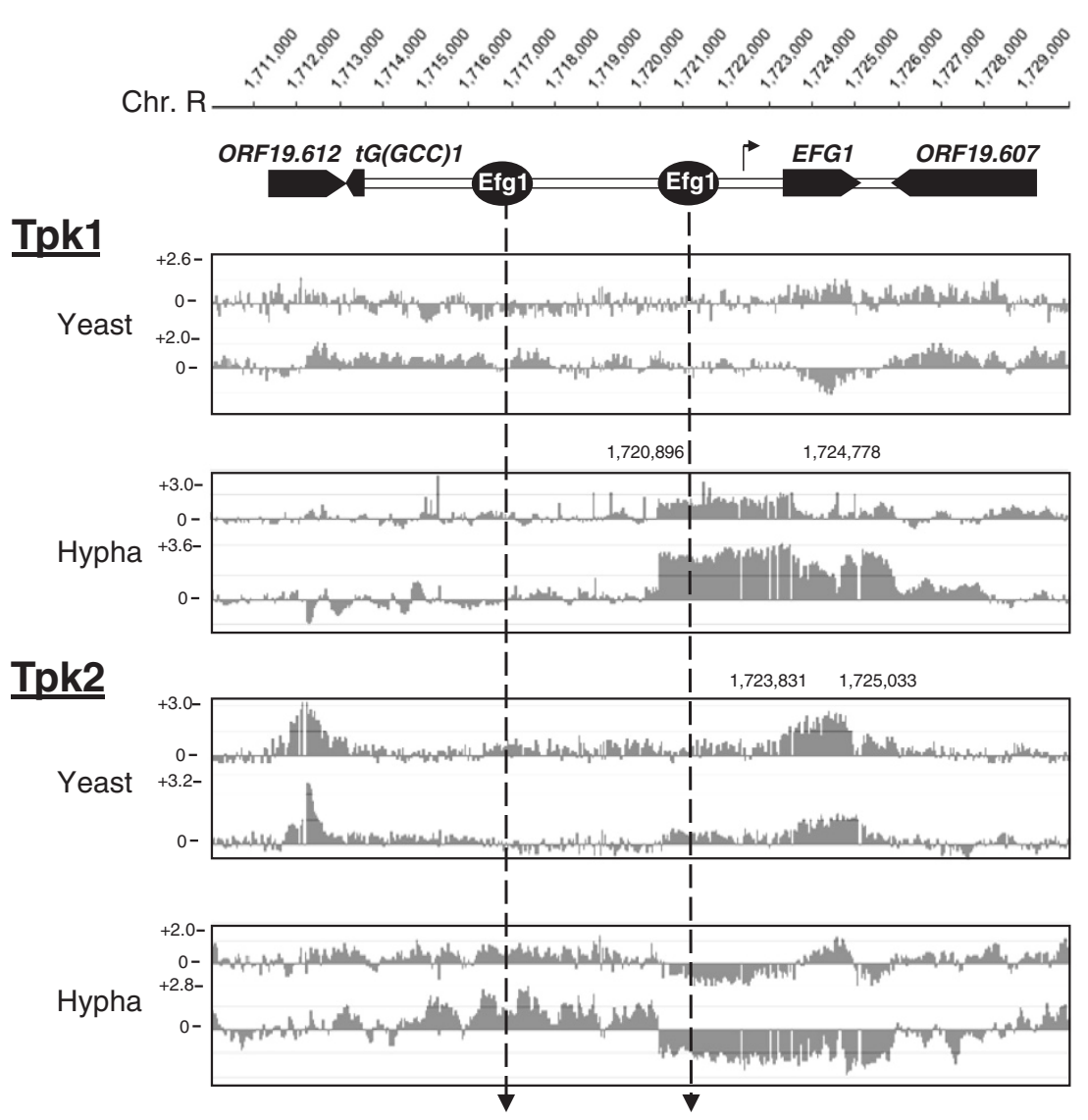

B

a) Yeast

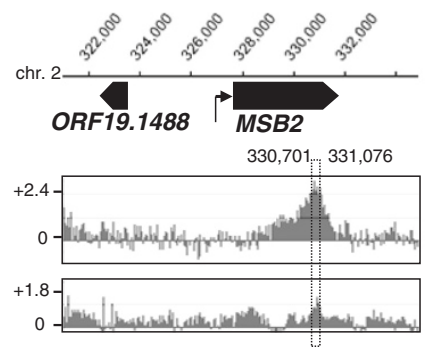

c) MSB2 transcript

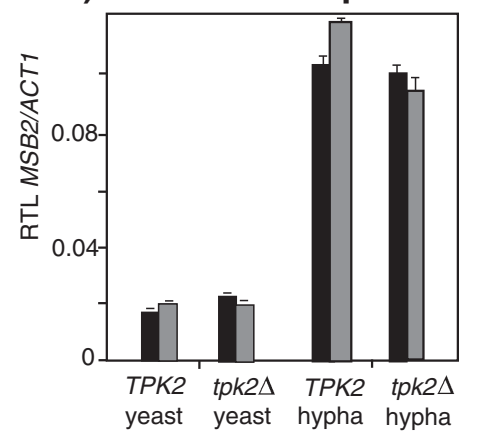

b) Hypha

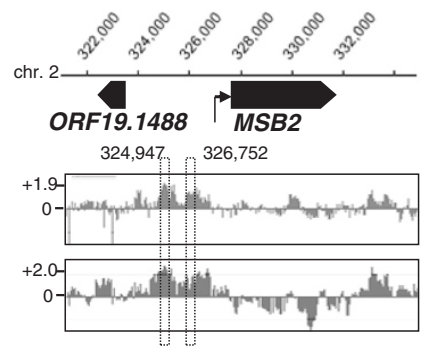

d) ORF19.1488 transcript

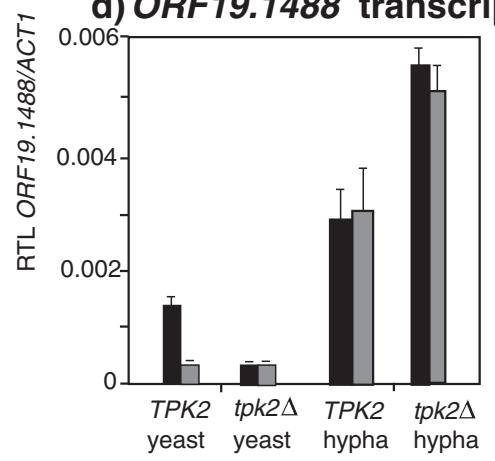

Figure 4 (See legend on next page.) 
(See figure on previous page.)

Figure 4 Tpk binding to EFG1 and MSB2 loci. A. Binding to EFG1. Coordinates of EFG1 and neighbouring genes on chromosome R are shown on top. Round circles (dotted lines) indicate the position of Efg1 binding during growth in the yeast form [13]; the kinked arrow indicates the transcript start site. The genomic localization of Tpk1 and Tpk2 was determined by ChIP chip assays on strains containing HA-tagged Tpk proteins relative to an unmarked control strain. For Tpk1 localization, the strain pair II (TPK1/tpk1)/AF1004 (TPK1-3× HA/tpk1) and for Tpk2 localization, the strain pair TPK7 (TPK2/tpk2)/AF1005 (TPK2-3× HA/tpk2) was used in ChIP chip. Graphs represent duplicates of Tpk ${ }^{\text {HA }}$ occupancy at the EFG1 locus, ordinates represent scaled $\log _{2}$ ratios. B. Binding to MSB2. Tpk2 enrichment in (a) the MSB2 ORF of yeast cells or (b) in the MSB2/ORF19.1488 upstream region of cells following hyphal induction; the most significant binding sequences are indicated by the dotted box. Transcript levels of MSB2 (c) or ORF19.1488 (d) in yeast or in induced cells were determined by qPCR in wild-type CAF2-1 (TPK2) or in tpk2 mutant cells (strain TPO7.4) growing in the yeast form or induced $30 \mathrm{~min}$ for hypha formation. Transcript levels relative to the ACT1 transcript (RTL) are shown for two biological replicates (black and grey bars) indicating means and standard deviations for three technical replicates.

During hyphal morphogenesis, binding of Tpk2 within promoter regions of several genes was correlated with transcript levels of these genes (Figure 5A-C). The transcript of the SOK1 gene (encoding a putative stressregulated kinase [32]) was downregulated during hyphal induction in wild-type strains, which did not occur but was even upregulated in a tpk2 mutant (Figure $5 \mathrm{~A}$ ). The Tpk2 binding peak (coordinates 1,271,612-1,272,002) lies directly upstream of the transcriptional start site of SOK1 at position 1,272,810 (Figure 5A, kinked arrow) [33]. During hyphal induction, Tpk2 binding in the intergenic region between ORF19.1488 and MSB2 genes did not affect the MSB2 transcript but correlated with an increased level of the ORF19.1488 transcript in the tpk2 mutant (Figure 4B). Furthermore, transcript upregulation of HYR1 and ECE1 genes, which are expressed specifically in the hyphal growth form $[34,35]$, was completely abolished in the $t p k 2 \Delta$ strain (Figure $5 \mathrm{~B}, \mathrm{C}$ ).

Promoter regions of SOK1, HYR1 and ECE1 genes are known to bind the Efg1 regulator relatively late during hyphal induction [26] or during biofilm formation [25], while no Efg1 binding was detected shortly (30 min) after hyphal induction [13]. Interestingly, Efg1 binding sequences are not identical but overlap partially with the

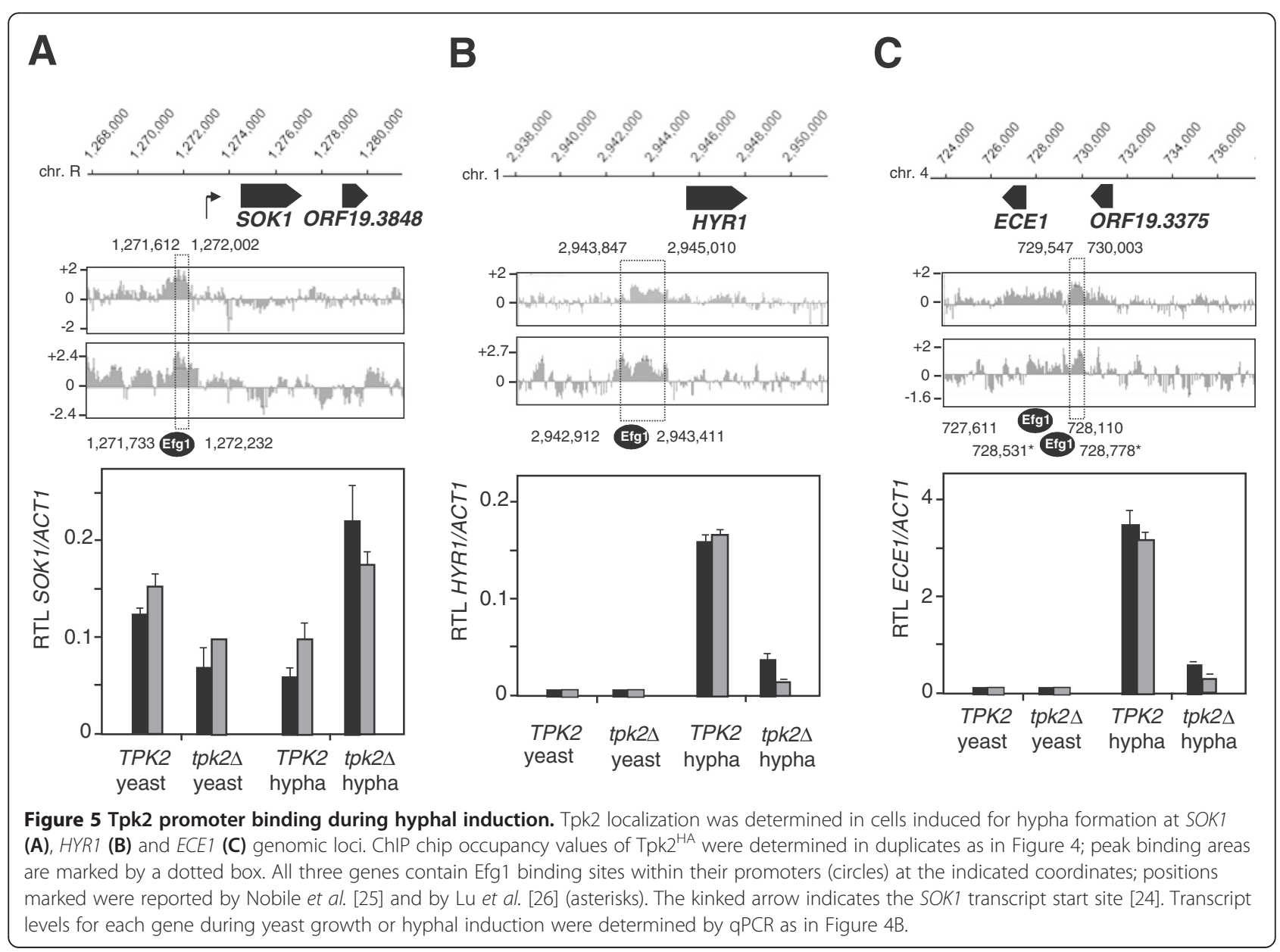


sequences bound by Tpk2 (Figure 5A-C). Taken together, these results suggest that Tpk2 binding to promoters has the potential to regulate transcription of $C$. albicans genes both negatively and positively, possibly involving subsequent binding of Efg1 as a PKA phosphorylation target [9].

\section{Sequence motifs in Tpk2 binding regions}

Sequences representing the binding peaks of PKA were analysed for consensus sequences using the RSAT programs dyad-analysis and peak-motifs [36,37]; both algorithms generated identical results. While no significant consensus motifs were detected for Tpk1, Tpk2 showed clear sequence preferences. If cells were grown in the yeast form, Tpk2 binding occurred most frequently within ORFs (Figure 2B) at ACCAC, CCACC or CAGC motifs (Figure 6A). During hyphal induction, however, when Tpk2 binds predominantly within promoter regions (Figure 2B), a completely different set of binding preferences was found (Figure 6A). The identified $\mathrm{A}_{5} \mathrm{GA}_{5}$ and $\mathrm{A}_{2} \mathrm{GA}_{5}$ motifs match the $\mathrm{A}_{2} \mathrm{GA}_{5}$ motif for binding of the Azf1 transcriptional regulator in S. cerevisiae, which is required for glucose-induced gene transcription [38]. Consistent with this activity, effective hypha formation in C. albicans is known to require low levels of glucose [39]. The identified AAC, AAG and ACC repeats, which were identified in most Tpk2 binding sites, had been also detected previously for the Efg1 transcriptional regulator during hyphal induction [13]. The HYR1 and ECE1 genes, which are induced by hypha formation [34,35], contain these consensus sequence motifs, possibly to permit binding of kinase and its downstream target Efg1 to jointly trigger morphogenesis-dependent gene expression.

Binding of Tpk2 to many ORFs raised the question if such bound ORF sequences were as free as unbound regions to evolve sequence variants, e. g. with regard to the usage of synonymous codons. Therefore, we compared overall C. albicans codon usage with codon usage in ORF sequences bound by Tpk2. Specifically, we investigated if codons corresponding to the deduced Tpk2 binding consensus sequences would be preferred in the Tpk2 binding region. It was indeed found that usage of all six codons matching the Tpk2 consensus sequence during yeast growth was increased as compared to the average codon usage in C. albicans or to random set of 150 ORFs ("out group") that are not bound by Tpk2 (Figure 6B). In the case of histidine even a complete reversal of codon usage from the preferred CAT codon (15.62/1000 to 8.63/1000) to CAC (5.39/1000 to $11.4 /$ 1000) was observed in the Tpk2 binding region. This result suggests that ORF binding Tpk2 has a vital, yet unknown function, because it exerts selective pressure to restrict codon usage within ORFs. Codon usage has hitherto been related mainly to abundance of aminacyltRNAs [40].

\section{PKA localization at the EFG1 locus}

The EFG1 gene is a paradigm of both PKA and Efg1 binding to a genomic locus (scheme of main events in Figure 7). Early during hyphal induction the EFG1 transcript level is lowered rapidly, presumably because the continued presence of Efg1 disturbs hyphal morphogenesis [29]. EFG1 downregulation requires the Efg1 protein and the Tpk2 PKA isoform [13] within a short time window to initiate hyphal formation, because both proteins leave the EFG1 locus rapidly thereafter. Possibly, genomic binding of both proteins prepares yeast cells to undergo hyphal morphogenesis rapidly in inducing environments. Binding of the Tpk1 isoform at promoter and coding region of EFG1, as well as binding of the Set3C histone deacetylase complex to the ORF [27] may also help to establish the repressed state of EFG1 during hyphal formation. EFG1 expression is probably regulated by additional proteins binding the EFG1 promoter directly or indirectly by adapter proteins including Efg1. Candidate Efg1-binding proteins localized to the EFG1 promoter include Czf1, Flo8, Sfl1, Sfl2 and Ndt80 proteins $[11,12,25]$. Tpk2 binding to the SET3 and SFL1 promoters, which we observed (Figure 3), may support this activity. Co-operation of Tpk2 and Efg1 may also occur at other genes regulated by hyphal induction including HYR1, ECE1 and SOK1 genes, as described above. Thus, the proposed Tpk2 phosphorylation of Efg1 $[9,13]$ and other components of the transcriptional initiation machinery could occur directly at the promoters of genes regulating morphogenesis.

\section{Conclusions}

PKA isoforms Tpk1 and Tpk2 are crucial for the virulence of the human fungal pathogen $C$. albicans by regulating dimorphic growth. Tpk proteins mediate environmental cues and trigger hyphal morphogenesis by altering the transcriptional program. We show that Tpk2 and to a lesser extent Tpk1 bind to specific genomic sequences within ORFs and promoters of target genes. Growth in the yeast form triggers Tpk binding to CA-rich sequences within ORFs and appears to bias codon usage within the binding region. During hyphal induction Tpk2 associates with promoter regions of genes regulating or regulated by hyphal morphogenesis, often proximal to binding sites for the Efg1 transcription factor. These results suggest that genomic PKA proteins facilitate and/or prolong hyphal morphogenesis by acting on nearby transcription factors at genes regulating morphogenesis. Molecular mechanisms of PKA nuclear import, genomic recruitment and function remain to be established. In conclusion, we have demonstrated for the first time in a fungal 
A

\section{Yeast}

\begin{tabular}{|c|c|c|c|}
\hline rank & sequence & E-value & P-value \\
\hline 1 & CACN $\{0\} C A C$ & $2.2 \mathrm{e}-50$ & $9.5 \mathrm{e}-45$ \\
\hline 2 & CAGN $\{3\} C A G$ & $3.2 \mathrm{e}-50$ & $1.4 \mathrm{e}-45$ \\
\hline 3 & ACCN $\{0\} A C C$ & $1.1 \mathrm{e}-49$ & $4.8 \mathrm{e}-45$ \\
\hline 4 & AGCN $\{0\} A G C$ & $2.0 \mathrm{e}-41$ & $8.6 \mathrm{e}-37$ \\
\hline 5 & CAGN $\{0\} C A G$ & $2.1 \mathrm{e}-39$ & $9.0 \mathrm{e}-35$ \\
\hline
\end{tabular}

ACCACC 166/204

CACCAC 149/204

ACCAC $\quad 345 / 204$

CCACC 231/204

CAGCA 172/204

AGCAG 120/204

CAGCAG 36/204

AGCAGC 37/204

CAGNNNCAG $\quad 127 / 204$

\section{Hypha}

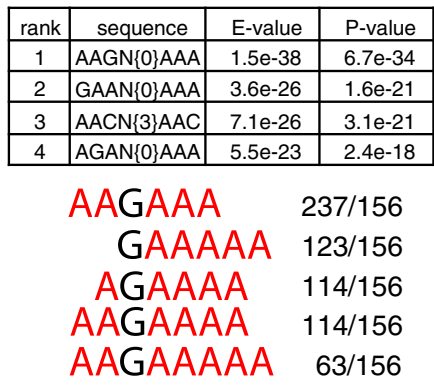

AACNNNAAC 40/156

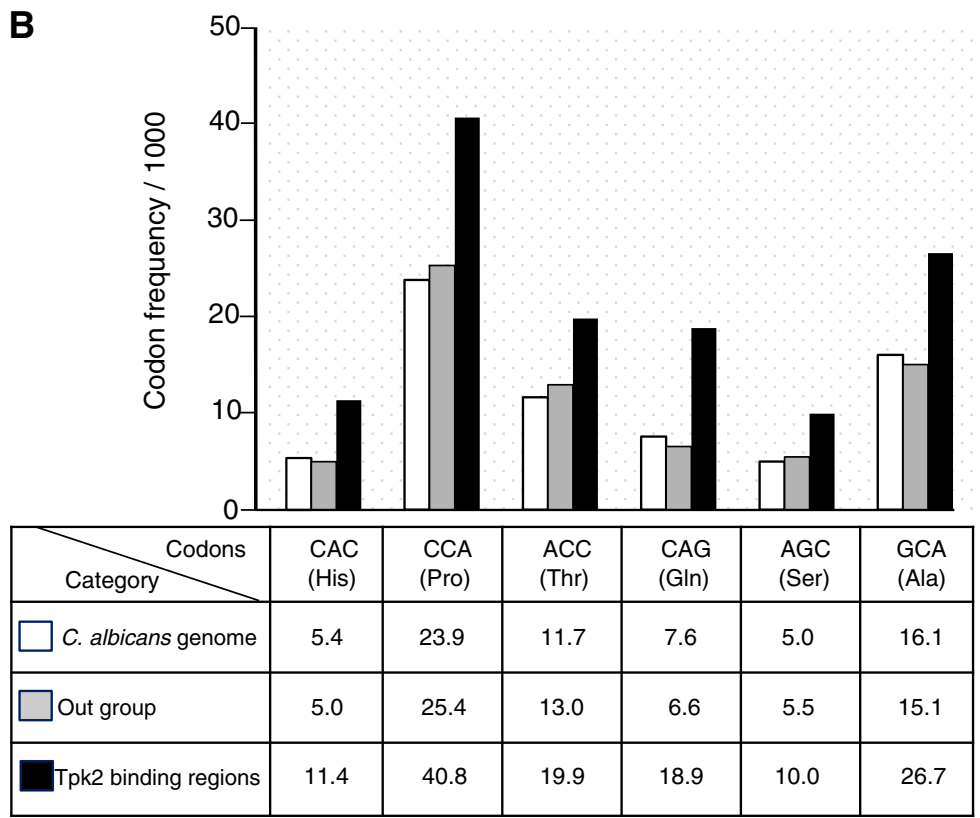

Figure 6 Tpk2 binding specificity. A. Consensus sequences for Tpk2 binding sites in yeast cells (left) or in cells induced for hypha formation (right). The program RSAT dyad-analysis [36] was used to predict consensus sequences. Predicted dyads (pairs of trinucleotides) common to Tpk2 binding sites were ranked and qualified by their $P$-/E-values; the frequencies of the deduced consensus sequences and of closely related sequences relative to all binding sites are listed below each table. B. Occurrence of trinucleotides/codons in ORF regions bound by Tpk2 during yeast growth. Codons corresponding to the Tpk2 binding consensus sequence are listed, along with codon frequencies in all C. albicans ORFs, in a randomly picked group of 150 genes not binding Tpk2 (out group) and in the 204 Tpk2 binding regions occurring mostly within ORFs.

pathogen that PKA isoforms, which are responsible for a relatively simple developmental program in a single cell, mark downstream target genes. Such binding analyses have predictive value because they link yet uncharacterized genes to signalling by PKA isoforms. This concept may hold true for other cellular differentiation processes involving other types of kinases and other species.

\section{Methods}

\section{Strains and growth conditions}

C. albicans strains are listed in Table 1 Strains were grown in liquid or on solid YPD, YPS and supplemented SD minimal medium, as described [13]. To induce hyphae the strains were grown at $37^{\circ} \mathrm{C}$ in YP medium containing $10 \%$ horse serum. C. albicans strains producing Cterminally HA-tagged PKA isoforms were constructed by 


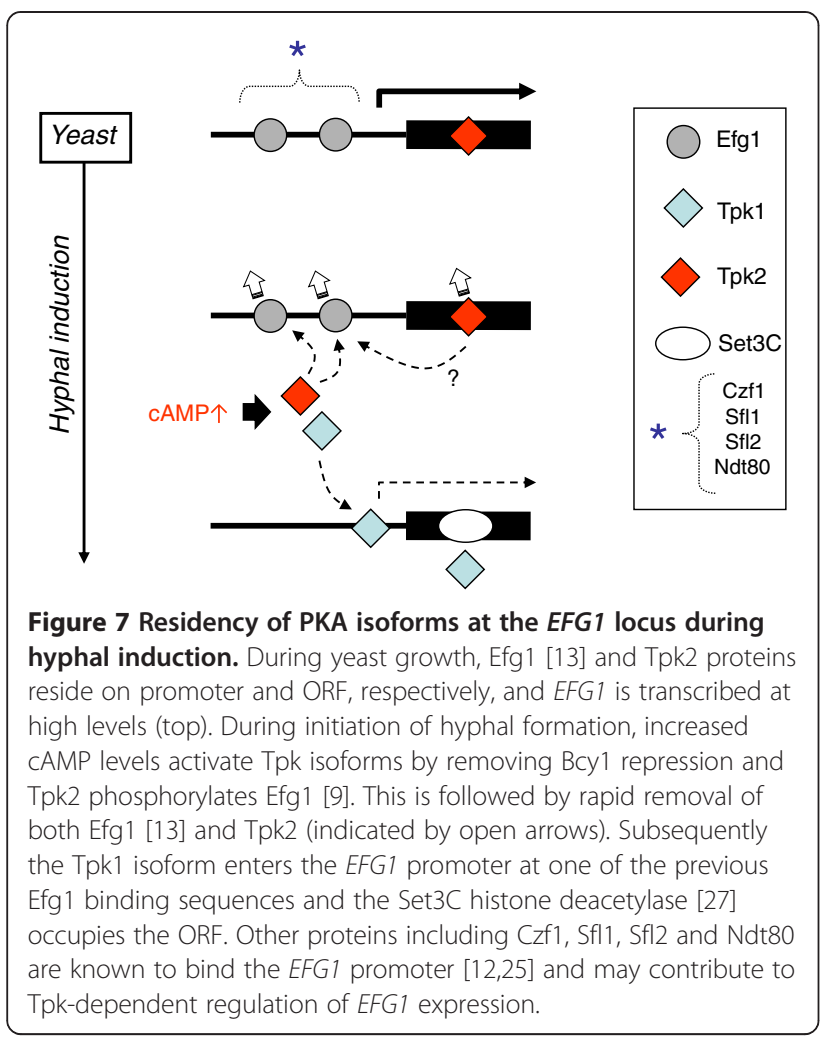

transformation of heterozygous strains retaining a single allele of the respective gene. HA-URA3 tagging cassettes were PCR amplified using oligonucleotide pairs on template plasmid P3HA-URA3, which generated PCR products ending in homologous sequences to target the respective genes [41]. Oligonucleotides Tpk1-HA (for)/(rev) and Tpk2-HA (for)/(rev) were used to generate tagged strains AF1003 $\left(T P K 1^{H A} / T P K 1\right)$ from CAI4, AF1004 (TPK1 $\left.{ }^{H A} / t p k 1\right)$ from FII4a and AF1005 (TPK2 $\left.{ }^{H A} / T P K 2\right)$ from AF1001. Similarly, oligonucleotides Bcy1-HA (for)/(rev) were used to tag one of the $B C Y 1$ alleles of strain CAI4 to generate strain AF1007 $\left(B C Y 1^{H A} / B C Y 1\right)$. Correct chromosomal integration of tagging cassettes was verified by colony PCR using primers TPK1ver, TPK2ver, BCY1ver in combination with primer 3'Test HA-tag. Oligonucleotides are listed in Additional file 2: Table S6.

\section{Immunodetection}

Proteins containing a hemagglutinin (HA) antigenic tag were detected in cell extracts by immunoblotting using monoclonal rat anti-HA antibody (Roche; 1:1000), which was visualized on blots using peroxidase-coupled goat antibody (Pierce; 1:10000). Cells used for immunofluorescence microscopy were fixed by $4 \%$ formaldehyde and $1 \mathrm{ml}$ of cell suspension were treated by zymolyase T100 $(100 \mu \mathrm{g})$, glucuronidase $(30 \mu \mathrm{l})$ and $10 \mathrm{mM}$ DTT for $30 \mathrm{~min}$ at $30^{\circ} \mathrm{C}$. Cells were pelleted and treated with $0.1 \%$ Triton X-100 for $5 \mathrm{~min}$ at room temperature. Cells $(20 \mu \mathrm{l})$ were fixed to polylysine-coated glass slides and washed with PBS, followed by blocking of unspecific binding sites using $2 \%$ milk powder in PBS. The blocking solution was removed and $40 \mu \mathrm{l}$ of rat anti-HA antibody (Roche; 1:100) were allowed to react $90 \mathrm{~min}$ at room temperature or overnight at $4{ }^{\circ} \mathrm{C}$ in a wet chamber. Cells were washed and fluorescein isothiocyanate (FITC)-coupled goat anti-rat antibody (Jackson Immunologic Research Lab Inc.; 1:100) in $0.2 \%$ milk powder was added and allowed to react for $90 \mathrm{~min}$ at room temperature. For nuclear staining $20 \mu \mathrm{l}$ diamidino-2-phenylindole (DAPI; $1 \mu \mathrm{g} / \mathrm{ml}$ ) was added for $15 \mathrm{~min}$ at room temperature. Slides were washed by PBS and a drop of anti-fade (Pro-Long Anti-Fade, Sigma) was added before covering the specimen with a cover slip, which was sealed by nail polish. Microscopic inspection of FITC and DAPI fluorescence was done using a spinning disc confocal microscope (Cell Observer SD; Yokogawa CSU-X1) and using the program Zen 2011 (Carl Zeiss) for evaluation of images.

\section{Chromatin immunoprecipitation on microchips (ChIP chip)}

The ChIP chip procedure was carried out essentially as described [13] except that magnetic beads with bound antibodies were eluted twice with elution buffer for $20 \mathrm{~min}$ at $65^{\circ} \mathrm{C}$ and that RNA was removed by adding $2.5 \mu \mathrm{l}$ of RNase A (10 mg/ml; Qiagen). C. albicans genomic tiling microarrays were probed pairwise by immunoprecipitated chromatin of a strain producing an HA-tagged protein and a corresponding control strain. The following pairs of strains were used: II (TPK1/tpk1)/AF1004 (TPK1-3× HA/tpk1), TPK7 (TPK2/tpk2)/AF1005 (TPK2-3× HA/tpk2), CAF2-1 (BCY1/BCY1)/AF1007 (BCY1-3× HA/BCY1). Two independent cultures were assayed for each combination of strains. Significant binding peaks were defined as probes containing four or more signals above background in a 500 bp sliding window; the degree of significance depended on the FDR value. Results were visualized using the

Table 1 C. albicans strains

\begin{tabular}{|c|c|c|}
\hline Strains & & Reference \\
\hline CAF2-1 & URA3/ura3::imm434 & {$[42]$} \\
\hline CAI4 & ura3::imm434/ura3::imm434 & [42] \\
\hline$\|$ & As CAI4 but TPK1/tpk1::hisG-URA3-hisG & [3] \\
\hline $\mathrm{F} \| 4 \mathrm{a}$ & As CAI4 but TPK1/tpk1::hisG & {$[3]$} \\
\hline AF1003 & As CAI4 but TPK1::(3xHA-URA3)/TPK1 & This work \\
\hline AF1004 & As Fll4a but TPK1::(3xHA-URA3)/tpk1::hisG & This work \\
\hline TPK7 & As CAl4 but TPK2/tpk2::hisG-URA3-hisG & {$[2]$} \\
\hline TPO7 (AF1001) & As TPK7 but TPK2/tpk2::hisG & {$[2]$} \\
\hline TPO7.4 & As CAl4 but tpk2::hisG/tpk2::hisG-URA3-hisG & {$[2]$} \\
\hline AF1005 & As AF1001 but TPK2::(3xHA-URA3)/tpk2::hisG & This work \\
\hline AF1007 & As CAI4 but $B C Y 1::(3 \times H A-U R A 3) / B C Y 1$ & This work \\
\hline
\end{tabular}


program SignalMap (version 1.9). The most significant binding peaks (FDR $\leq 0.05$ ), which coincided in both replicates, were analyzed by the program RSAT dyad-analysis to predict binding sequence from all peak genomic binding sites [36]. Codon usage of all C. albicans genes was derived from the Candida Genome Database [24] and codon usage in sequences of ORFs bound by Tpk2 were calculated using the Codon Usage Calculator [43].

\section{Availability of supporting data}

The data sets supporting the results of this article are available in the Candida Genome Database (CGD) repository: http://www.candidagenome.org/download/systematic_ results/Schaekel_2013/.

\section{Additional files}

Additional file 1: Table S1. Lists Tpk1 binding sites during yeast growth. Table S2. Lists Tpk1 binding sites during hyphal induction. Table S3. Lists Tpk2 binding sites during yeast growth. Table S4. Lists Tpk2 binding sites during hyphal induction. Table S5. Lists Bcy1 binding sites during yeast growth.

Additional file 2: Table S6. List of oligonucleotides.

\section{Competing interests}

The authors declare that they have no competing interests.

\section{Authors' contributions}

AS did the experiments. AS and PD did the bioinformatics analysis. JFE did the experimental design and wrote the paper. All authors have read and approved the manuscript for publication.

\section{Acknowledgements}

We thank the members of the CAI center, Heinrich-Heine-Universität Düsseldorf, for assistance in microscopy. We thank K. Sanyal for helpful discussions. This project was supported by the Jürgen Manchot Stiftung Düsseldorf, by the Deutsche Forschungsgemeinschaft (ER47.13-1) and by ERA-NET PathoGenoMics project OXYstress.

Received: 3 June 2013 Accepted: 15 November 2013 Published: 1 December 2013

\section{References}

1. Sudbery PE: Growth of Candida albicans hyphae. Nat Rev Microbio/ 2011, 9:737-748.

2. Sonneborn A, Bockmühl DP, Gerads M, Kurpanek K, Sanglard D, Ernst JF: Protein kinase $A$ encoded by TPK2 regulates dimorphism of the human pathogen Candida albicans. Mol Microbiol 2000, 35:386-396.

3. Bockmühl DP, Krishnamurthy S, Gerads M, Sonneborn A, Ernst JF: Distinct and redundant roles of the two protein kinase $A$ isoforms Tpk1p and Tpk2p in morphogenesis and growth of Candida albicans. Mol Microbiol 2001, 42:1243-1257.

4. Giacometti R, Kronberg F, Biondi RM, Passeron S: Catalytic isoforms Tpk1 and Tpk2 of Candida albicans PKA have non-redundant roles in stress response and glycogen storage. Yeast 2009, 26:273-285.

5. Cassola A, Parrot M, Silberstein S, Magee BB, Passeron S, Giasson L, Cantore ML: Candida albicans lacking the gene encoding the regulatory subunit of protein kinase A displays a defect in hyphal formation and an altered localization of the catalytic subunit. Eukaryot Cell 2004, 3:190-199.

6. Harcus D, Nantel A, Marcil A, Rigby T, Whiteway M: Transcription profiling of cyclic AMP signaling in Candida albicans. Mol Biol Cell 2004, 15:4490-4499.

7. Hall RA, Mühlschlegel FA: A multi-protein complex controls CAMP signalling and filamentation in the fungal pathogen Candida albicans. Mol Microbiol 2010, 75:534-537.
8. Stoldt VR, Sonneborn A, Leuker C, Ernst JF: Efg1, an essential regulator of morphogenesis of the human pathogen Candida albicans, is a member of a conserved class of bHLH proteins regulating morphogenetic processes in fungi. EMBO J 1997, 16:1982-1991.

9. Bockmühl DP, Ernst JF: A potential phosphorylation site for an A-type kinase in the Efg1 regulator protein contributes to hyphal morphogenesis of Candida albicans. Genetics 2001, 157:1523-1530.

10. Doedt T, Krishnamurthy S, Bockmühl DP, Tebarth B, Stempel C, Russell CL, Brown AJ, Ernst JF: APSES proteins regulate morphogenesis and metabolism in Candida albicans. Mol Biol Cell 2004, 15:3167-3180.

11. Noffz CS, Liedschulte $V$, Lengeler K, Ernst JF: Functional mapping of the Candida albicans Efg1 regulator. Eukaryot Cell 2008, 7:881-893.

12. Znaidi S, Nesseir A, Chauvel M, Rossignol T, D'Enfert C: A Comprehensive functional portrait of two heat shock factor-type transcriptional regulators involved in Candida albicans morphogenesis and virulence. PLOS Pathog 2013, 9:e1003519.

13. Lassak T, Schneider E, Bussmann M, Kurtz D, Manak JR, Srikantha T, Soll DR Ernst JF: Target specificity of the Candida albicans Efg1 regulator. Mol Microbiol 2011, 82:602-618.

14. Griffioen G, Anghileri P, Imre E, Baroni MD, Ruis H: Nutritional control of nucleocytoplasmic localization of cAMP-dependent protein kinase catalytic and regulatory subunits in Saccharomyces cerevisiae. J Biol Chem 2000, 275:1449-1456.

15. Matsuo $Y$, Mclnnis B, Marcus S: Regulation of the subcellular localization of cyclic AMP-dependent protein kinase in response to physiological stresses and sexual differentiation in the fission yeast Schizosaccharomyces pombe. Eukaryot Cell 2008, 7:1450-1459.

16. Beene DL, Scott JD: A-kinase anchoring proteins take shape. Curr Opin Cell Biol 2007, 19:192-198.

17. Sastri M, Barraclough DM, Carmichael PT, Taylor SS: A-kinase-interacting protein localizes protein kinase $A$ in the nucleus. Proc Natl Acad Sci USA 2005, 102:349-354.

18. Ernst JF, Bockmühl DP: Gene expression and genetic techniques. In Candida and Candidiasis. Edited by Calderone R. Washington: ASM Press; 2001:267-278

19. Pokholok DK, Zeitlinger J, Hannett NM, Reynolds DB, Young RA: Activated signal transduction kinases frequently occupy target genes. Science 2006, 313:533-536.

20. Cook KE, O'Shea EK: Hog1 controls global reallocation of RNA Polll upon osmotic shock in Saccharomyces cerevisiae. G3 (Bethesda) 2012, 2:1129-1136.

21. Nadal-Ribelles M, Conde N, Flores O, González-Vallinas J, Eyras E, Orozco M, De Nadal E, Posas F: Hog1 bypasses stress-mediated down-regulation of transcription by RNA polymerase II redistribution and chromatin remodeling. Genome Biol 2012, 3:R106.

22. Pascual-Ahuir A, Proft M: The Sch9 kinase is a chromatin-associated transcriptional activator of osmostress-responsive genes. EMBO J 2007 26:3098-3108.

23. Zheng X, Wang Y, Wang Y: Hgc1, a novel hypha-specific G1 cyclin-related protein regulates Candida albicans hyphal morphogenesis. EMBO J 2004, 23:1845-1856.

24. Arnaud MB, Costanzo MC, Skrzypek MS, Binkley G, Lane C, Miyasato SR, Sherlock G: The Candida Genome Database (CGD), a community resource for Candida albicans gene and protein information. Nucleic Acids Res 2005, 33:D358-D363. http://www.candidagenome.org/.

25. Nobile CJ, Fox EP, Nett JE, Sorrells TR, Mitrovich QM, Hernday AD, Tuch BB, Andes DR, Johnson AD: A recently evolved transcriptional network controls biofilm development in Candida albicans. Cell 2012, 148:126-138.

26. Lu Y, Su C, Mao X, Raniga PP, Liu H, Chen J: Efg1-mediated recruitment of NuA4 to promoters is required for hypha-specific Swi/Snf binding and activation in Candida albicans. Mol Biol Cell 2008, 19:4260-4272.

27. Hnisz D, Bardet AF, Nobile CJ, Petryshyn A, Glaser W, Schöck U, Stark A, Kuchler K: A histone deacetylase adjusts transcription kinetics at coding sequences during Candida albicans morphogenesis. PLoS Genet 2012, 8:e1003118.

28. Nantel A, Dignard D, Bachewich C, Harcus D, Marcil A, Bouin AP, Sensen CW, Hogues H, Van het Hoog M, Gordon P, Rigby T, Benoit F, Tessier DC, Thomas DY, Whiteway M: Transcription profiling of Candida albicans cells undergoing the yeast-to-hyphal transition. Mol Biol Cell 2002, 13:3452-65. 
29. Tebarth B, Doedt T, Krishnamurthy S, Weide M, Monterola F, Dominguez A Ernst JF: Adaptation of the Efg1p morphogenetic pathway in Candida albicans by negative autoregulation and PKA-dependent repression of the EFG1 gene. J Mol Biol 2003, 329:949-962.

30. Román E, Cottier F, Ernst JF, Pla J: Msb2 signaling mucin controls activation of Cek1 mitogen-activated protein kinase in Candida albicans. Eukaryot Cell 2009, 8:1235-1249.

31. Szafranski-Schneider E, Swidergall M, Cottier F, Tielker D, Román E, Pla J, Ernst JF: Msb2 shedding protects Candida albicans against antimicrobial peptides. PLoS Pathog 2012, 8:e1002501.

32. Bennett RJ, Johnson AD: The role of nutrient regulation and the Gpa2 protein in the mating pheromone response of $C$. albicans. Mol Microbiol 2006, 62:100-119.

33. Tuch BB, Mitrovich QM, Homann OR, Hernday AD, Monighetti CK, De La Vega FM, Johnson AD: The transcriptomes of two heritable cell types illuminate the circuit governing their differentiation. PLOS Genet 2010, 6:e1001070.

34. Birse CE, Irwin MY, Fonzi WA, Sypherd PS: Cloning and characterization of $E C E 1$, a gene expressed in association with cell elongation of the dimorphic pathogen Candida albicans. Infect Immun 1993, 61:3648-3655.

35. Bailey DA, Feldmann PJ, Bovey M, Gow NA, Brown AJ: The Candida albicans HYR1 gene, which is activated in response to hyphal development, belongs to a gene family encoding yeast cell wall proteins. J Bacteriol 1996, 178:5353-53560.

36. Van Helden J, Rios AF, Collado-Vides J: Discovering regulatory elements in non-coding sequences by analysis of spaced dyads. Nucleic Acids Res 2000 28:1808-1818.

37. Thomas-Chollier M, Herrmann C, Defrance M, Sand O, Thieffry D, Van Helden J: RSAT peak-motifs: motif analysis in full-size ChIP-seq datasets. Nucleic Acids Res 2012, 40:e31.

38. Newcomb LL, Hall DD, Heideman W: AZF1 is a glucose-dependent positive regulator of CLN3 transcription in Saccharomyces cerevisiae. Mol Cell Biol 2002, 22:1607-1614.

39. Hudson DA, Sciascia QL, Sanders RJ, Norris GE, Edwards PJ, Sullivan PA, Farley PC: Identification of the dialysable serum inducer of germ-tube formation in Candida albicans. Microbiology 2004, 150:3041-3049.

40. Novoa EM, Ribas de Pouplana L: Speeding with control: codon usage, tRNAs, and ribosomes. Trends Genet 2012, 28:574-581.

41. Prill SK, Klinkert B, Timpel C, Gale CA, Schröppel K, Ernst JF: PMT family of Candida albicans: five protein mannosyltransferase isoforms affect growth, morphogenesis and antifungal resistance. Mol Microbio/ 2005, 55:546-560.

42. Fonzi WA, Irwin MY: Isogenic strain construction and gene mapping in Candida albicans. Genetics 1993, 134:717-728.

43. Codon Usage Calculator. http://geneinfinity.org/sms/sms_codonusage.html.

doi:10.1186/1471-2164-14-842

Cite this article as: Schaekel et al: Morphogenesis-regulated localization of protein kinase A to genomic sites in Candida albicans. BMC Genomics $201314: 842$.

\section{Submit your next manuscript to BioMed Central and take full advantage of:}

- Convenient online submission

- Thorough peer review

- No space constraints or color figure charges

- Immediate publication on acceptance

- Inclusion in PubMed, CAS, Scopus and Google Scholar

- Research which is freely available for redistribution 